\title{
Photocatalytic Degradation of Deoxynivalenol using Graphene/ZnO Hybrids in Aqueous Suspension
}

\author{
Xiaojuan Bai ${ }^{a^{*}}$, Changpo Sun ${ }^{a^{*}}$, Di Liu ${ }^{b}$, Xiaohong Luo ${ }^{a}, \mathrm{Di}_{\mathrm{Li}}^{c}$, \\ Jun Wang ${ }^{a}$, Nanxi Wang ${ }^{a}$, Xiaojiao Chang ${ }^{a}$, Ruilong Zong ${ }^{d}$, \\ Yongfa $\mathrm{Zhu}^{d}$
}

\begin{abstract}
:
Water-soluble deoxynivalenol (DON) pose a major threat as a potential organic pollutant to water environmental quality. DON is a toxic secondary metabolite produced by molds of the Fusarium genus and one of the most important mycotoxins in cereal commodities, which can be enriched from the contaminated grain by deoxynivalenol in the process of wet processing. In this work, graphene/ZnO hybrids has been successfully prepared via a simple one-step hydrothermal method and exhibited superior photocatalytic activity for the photodegradation of DON under the irradiation of UV light. The UV light photocatalytic activity of graphene/ZnO hybrid GZ0.3 was 3.1 times than pure $\mathrm{ZnO}$ and about $99 \%$ of DON (15 ppm) could be photodegraded within 30 min totally while there were three peaks of intermediate products appeared. The ESI/MS analysis confirmed the presence of DON and degradation product with the secondary mass spectrogram in the positive ESI mode. Overall, this work could provide new insights into the fabrication of graphene/ZnO hybrids composite as high performance photocatalysts and facilitate their application in the mycotoxin detoxification and environmental protection issues.
\end{abstract}

Keywords: photocatalysis; $\mathrm{ZnO}$; graphene; hybridization; deoxynivalenol 


\section{Introduction}

Water-soluble deoxynivalenol pose a major threat as a potential organic pollutant to water environmental quality. In the process of wet processing, soluble-toxin molecules can be enriched from the contaminated grain by deoxynivalenol, which will pose a potentially serious threat to water environment. Deoxynivalenol belongs to trichothecenes which are a group of over 180 structurally related sesquiterpenoid mycotoxins produced mainly by Fusarium species growing on basic commodities including wheat, maize and barley. ${ }^{1-2}$ The group is subdivided in type A trichothecenes such as T-2 toxin and type B trichothecenes such as deoxynivalenol, the difference being a keto substitution at C-8 in the latter case (Figure S1). ${ }^{3}$ Although type B trichothecenes are generally less acutely toxic than type A-, C-, and D-trichothecenes, they are the most prevalent congeners in grain. ${ }^{4}$ DON possesses structural and toxicity features that may give rise to chemical reactivity-the epoxyring, hydroxyl groups, and an $\alpha, \beta$-unsaturated carbonyl group. ${ }^{5,6}$ Bretz et al reported that the stability of DON under food-processing conditions such as cooking or baking and performed model heating experiments to screen the residue for degradation products. They found that heating of DON and 3-acetyldeoxynivalenol (3-AcDON) can give a mixture of compounds under alkaline conditions and reduce the toxicity of DON. ${ }^{3}$ Park et al investigated the degradation of three different mycotoxins, aflatoxin B1 (AFB1), deoxynivalenol (DON) and nivalenol (NIV) by using our self-designed microwave-induced argon plasma system at atmospheric pressure. The mycotoxins AFB1, DON, and NIV were completely removed after $5 \mathrm{~s}$ of plasma treatment and the 
cytotoxicity of mycotoxins was significantly reduced with the progress in the treatment time. ${ }^{7}$ The $\gamma$-irradiation of AFB1 was reported to have encouraging degradation rates in several studies, ${ }^{8-11}$ and the identification of radiolytic products and the confirmation of toxicity have been studied by Wang et al group. ${ }^{12}$ Mishra et al studied influence of temperature and $\mathrm{pH}$ on the degradation of deoxynivalenol (DON) in aqueous medium and the results suggest that standard DON was unstable at $125-250{ }^{\circ} \mathrm{C}$ showing $16-100 \%$ degradation whereas DON at $\mathrm{pH} 1-3$ had 30-66 \% degradation, with a concomitant increase in the formation of a degraded product. ${ }^{13} \mathrm{Wu}$ et al performed systematic studies of a range of physicochemical parameters, such as temperature, moisture, compression, residence time in the extruder, $\mathrm{pH}$ value, and protein content, and it was shown that the reduction of deoxynivalenol levels is dependent on a set of parameters partially interacting with each other during extrusion cooking. ${ }^{14}$ To date, although numerous conventional physical, chemical and biological detoxification methods have been tested, none really fulfills the necessary efficacy and safety. Meanwhile, the degradation effect is limited and mechanism is still not clear, which needs further investigated deeply. Therefore, the development of a green and efficient detoxification technology is essential to improve the efficiency of decontamination of soluble deoxynivalenol and reduce treatment cost. ${ }^{15-16}$

The photocatalytic degradation technology is gaining importance in the area of pollutants treatment, especially for wastewater containing small amounts of refractory organic substances, which could be applied for mycotoxin detoxification. The technology has several advantages over competing methods. These are: (1) complete 
mineralization, (2) no waste disposal problem, (3) low cost, and (4) only mild temperature and pressure conditions are necessary. ${ }^{17}$ Although photocatalytic technology showed great potential for degradation of organic pollutants, it is still challenging to obtain high photocatalytic efficiency. Recombination of photoinduced charge is a key barrier for quantum efficiency of photocatalysis. ${ }^{18}$ Therefore, there is a great demand for a novel approach to promote the photocatalytic efficiency by decreasing the recombination rate. Graphene sheets (GS), excellent electron collectors and transporters, have been used to boost performance of various energy conversion and storage devices such as photovoltaic devices, supercapacitors, fuel cells, and Li-ion batteries. ${ }^{19-24} \mathrm{ZnO}$ semiconductor is an important photocatalyst due to its nontoxic nature, low cost, and high reactivity. It has been widely used in degradation of environmental pollutants in air or water, as well as transformation selective organic pollutants to nontoxic small molecule even to $\mathrm{CO}_{2} / \mathrm{H}_{2} \mathrm{O} \cdot{ }^{25,26}$ Recently, functionalized graphene-based semiconductor photocatalysts have attracted a lot of attention due to their good electron conductivity, large specific surface area and high adsorption. ${ }^{20}$ Although it has been shown previously that the graphene-based semiconductor exhibited enhanced photocatalytic performance than pure photocatalyst alone, some problems still hinder further application of the present nanocomposites.

In this work, it is the first time to demonstrate the graphene/ $\mathrm{ZnO}$ hybrid photocatalysts can be used to detoxification of DON in aqueous suspension and the probable structure of intermediate product is investigated as well as the influence of graphene on the photocatalytic activity for DON degradation has been elucidated 
systematically. Furthermore, the UV (254 nm, $365 \mathrm{~nm})$ light activity of $\mathrm{ZnO}$ for photodegradation of DON was enhanced after graphene surface hybridization. It was shown that the photocatalytic activity of $\mathrm{ZnO}$ /graphene hybrid for DON degradation is dependent on the content of graphene and exhibited stable efficiency toward the DON photodegradation for an extended period of time. The photocatalytic activity and intermediate product for DON degradation may provide new insight for green and efficient detoxification technology to improve the efficiency of decontamination of mycotoxins and reduce treatment cost.

\section{Experimental Section}

\section{Materials}

Graphite powder (325 mesh) were purchased from Qingdao Huatai Lubricant Sealing S\&T Co. Ltd., Qingdao, China. Acetonitrile and methanol of HPLC grade were obtained from Honeywell Co. Ltd. ZnO nanopowder (particle diameter 10-20 nm, surface area $10.4 \mathrm{~m}^{2} \mathrm{~g}^{-1}$ ) were commercially available. Water used in the work was purified with a Milli-Q system (Millipore, Billerica, MA). Potassium permanganate $\left(\mathrm{KMnO}_{4}\right)$, hydrochloric acid $(\mathrm{HCl})$, sulfuric acid $\left(\mathrm{H}_{2} \mathrm{SO}_{4}, 98 \%\right)$, hydrogen peroxide $\left(\mathrm{H}_{2} \mathrm{O}_{2}, 30 \%\right)$, phosphoric acid $\left(\mathrm{H}_{3} \mathrm{PO}_{4}, 85 \%\right)$ and trifluoroacetic acid (TFA) were from Sinopharm Chemical Reagent Co. Ltd. (Shanghai, China). All the chemicals and solvents were of analytical grade except acetonitrile and methanol, which were of HPLC grade. DON standard was purchased from Sigma-Aldrich (St. Louis, MO, USA). All the standard solutions were stored in the dark at $4{ }^{\circ} \mathrm{C}$.

\section{Synthesis of Graphene Oxide}


Graphene oxide was prepared by oxidation of natural graphite powder according to the modified Hummers' method. ${ }^{27}$ Briefly, graphite $(3.0 \mathrm{~g})$ was added to concentrated sulfuric acid $(70 \mathrm{~mL})$ under stirring at room temperature, then sodium nitrate $(1.5 \mathrm{~g})$ was added, and the mixture was cooled to $0{ }^{\circ} \mathrm{C}$. Under vigorous agitation, potassium permanganate $(9.0 \mathrm{~g})$ was added slowly to keep the temperature of the suspension lower than $20{ }^{\circ} \mathrm{C}$. Successively, the reaction system was transferred to a $35 \sim 40{ }^{\circ} \mathrm{C}$ water bath for about $0.5 \mathrm{~h}$, forming a thick paste. Then, $140 \mathrm{~mL}$ of water was added, and the solution was stirred for another $15 \mathrm{~min}$. An additional $500 \mathrm{~mL}$ of water was added followed by a slow addition of $20 \mathrm{~mL}$ of $\mathrm{H}_{2} \mathrm{O}_{2}(30 \%)$, turning the color of the solution from brown to yellow. The mixture was filtered and washed with 1:10 $\mathrm{HCl}$ aqueous solution $(250 \mathrm{~mL})$ to remove metal ions followed by repeated washing with water and centrifugation to remove the acid. The resulting solid was dispersed in water by ultrasonication for $1 \mathrm{~h}$ to make a graphene oxide aqueous dispersion $(0.5 \mathrm{wt} \%)$. The obtained brown dispersion was then subjected to $30 \mathrm{~min}$ of centrifugation at $4000 \mathrm{rpm}$ to remove any aggregates. Finally, it was purified by dialysis for 1 week to remove the remaining salt impurities for the following experiments.

\section{Preparation of Graphene-ZnO Hybrid Photocatalyst}

The graphene/ZnO hybrids were synthesized and the detail process as follows: Appropriate GO was well dispersed in distilled water, and then the disperstion was ultrasonicated for $60 \mathrm{~min}$ to get GO exfoliated. The obtained brown dispersion was then subjected to $30 \mathrm{~min}$ of centrifugation at low speed $5000 \mathrm{rpm}$ to remove any unexfoliated GO. Subsequently, the suspension was then centrifuged at high speed 
$10000 \mathrm{rmp}$ to remove the residual supernatant. The obtained exfoliated GO was then dispersed in $100 \mathrm{~mL}$ of water, and certain amount of pure $\mathrm{ZnO}$ was added into the GO dispersion, respectively. The $\mathrm{ZnO}$ nanoparticles and $\mathrm{GO}$ mixture was dispersed by ultrasonication for $30 \mathrm{~min}$ and stirred for $3 \mathrm{~h}$ and then homogeneous mixture dispersion was sealed in a $16 \mathrm{~mL}$ Teflon-lined autoclave and maintained at $180{ }^{\circ} \mathrm{C}$ for $6 \mathrm{~h}$. Then the autoclave was naturally cooled to room temperature and the as-prepared samples were taken out, washed with deionized water and ethanol in turn to remove surface impurities for following experiments. The graphene oxide content in the composite was tuned by changing the amount of the added graphite oxide. The graphene/ZnO hybrids with different mass ratio of graphene from $0.1 \%$ to $10.0 \%$ were prepared according to above method. The graphene/ZnO-X wt \% hybrid photocatalysts were marked as GZX, $\mathrm{X}$ label as graphene/ZnO hybrids mass ratio $0.1,0.3,3.0,5.0,8.0$, and 10.0.

\section{Characterizations}

The samples were characterized by powder X-ray diffraction (XRD) on a Bruker D8-advance X-ray diffractometer at $40 \mathrm{kV}$ and $40 \mathrm{~mA}$ for monochromatized $\mathrm{Cu} \mathrm{K} \alpha(\lambda$ $=1.5406 \AA$ ) radiation. The Brunauer-Emmett-Teller (BET) specific surface area of the samples was characterized by nitrogen adsorption at $77 \mathrm{~K}$ with a Micromeritics 3020 instrument. Zeta potential measurements were made with a Delsa Nano C zeta potential instrument (Beckman Coulter). The morphologies and sizes of the as-prepared products were examined through a LEO-1530 field emission Scanning Electron Microscope (SEM) and a HITACHI HT7700 transmission electron microscope (TEM). Fourier transform infrared spectra (FT-IR) were taken with a Bruker VERTEX 700 
spectrometer in the frequency range of $4000-600 \mathrm{~cm}^{-1}$ with a resolution of $4 \mathrm{~cm}^{-1}$. The diffuse reflectance absorption spectra (DRS) of the samples were recorded in the range from 250 to $800 \mathrm{~nm}$ using a Hitachi U-3010 spectroscope equipped with an integrated sphere attachment and $\mathrm{BaSO}_{4}$ was used as a reference. Raman spectra were recorded on a microscopic confocal Raman spectrometer (Renishaw $1000 \mathrm{NR}$ ) with an excitation of $514.5 \mathrm{~nm}$ laser light. The HPLC experiments were performed on an Waters 2695 Series LC system (Waters, Empower 3 2695-2489), which consisted of an autosampler, an online degasser, a quatpump, a thermostatted column compartment, and a fluorescence detector $\left(E_{x}=274 \mathrm{~nm}, E_{m}=440 \mathrm{~nm}\right)$. Waters ChemStation was employed for the LC system to acquire and analyze chromatographic data. Separation was performed on a C18 column ( $5 \mu \mathrm{m}$ particlesize, $150 \mathrm{~mm} \times 4.6 \mathrm{~mm}$ xbridge $)$ from Waters Co., Ltd. Thermogravimetric analysis (TGA) and differential thermal analysis (DSC) were performed on a Dupont 1090 thermal analyzer. The atmosphere was air and the heating rate was $10{ }^{\circ} \mathrm{C} \mathrm{min}^{-1}$. The MS and MS/MS analysis were performed on an Agilent Q-TOF 6510 time of flight mass spectrometry.

\section{Photocatalytic Experiments}

In this work, photo-degradation of organic pollutant deoxynivalenol (DON) in aqueous solution under UV ( $254 \mathrm{~nm}, 365 \mathrm{~nm})$ light irradiation was adopted to evaluate the photocatalytic performance of the graphene/ZnO photocatalysts $\operatorname{GZX}(0.1,0.3,3.0$, 5.0, 8.0, and 10.0). The concentration of deoxynivalenol (DON) is adjusted at $15 \mathrm{ppm}$. Before the light irradiation, a suspension of a $50 \mathrm{ml}$ probe pollutants solution and $25 \mathrm{mg}$ photocatalysts was stirred for $1 \mathrm{~h}$ under dark to reach the adsorption-desorption 
equilibrium. At certain time intervals, a suspension (2 $\mathrm{mL})$ was extracted and centrifugated to remove the photocatalyst. The concentrations of probe pollutants were characterized by HPLC system (Waters 2695 Series, LC system Waters, Empower 3 2695-2489) with a C18 column (5 $\mu$ m particlesize, $150 \mathrm{~mm} \times 4.6 \mathrm{~mm}$ xbridge) from Waters Co., Ltd reversed phase column.

\section{Mass spectrometry by ESI-MS}

ESI-MS studies were carried out to determine $\mathrm{m} / \mathrm{z}$ values for the degraded product of DON and DON standard solution. The electron spray MS and MS/MS spectra for HPLC eluate were recorded on an Agilent Q-TOF 6510 mass spectrometer equipped with a dual electron spray ionisation source. The ESI interface was used in positive ion mode with the following settings: gas temp at $350{ }^{\circ} \mathrm{C}$; drying gas with $10 \mathrm{~L} / \mathrm{min}$; pressure of nebulizer at $40 \mathrm{psig}$; fragmentor at $120 \mathrm{~V}$; skimmer at $65 \mathrm{~V}$; oCT $1 \mathrm{RF}$ Vpp at $750 \mathrm{~V}$; stop time about No limit/as pump Cycle Time at $0.333 \mathrm{~s}$. All other source parameters were automatically tuned for maximum signal intensity of a reference solution (Agilent). The mass spectrometer was operated in a scan range from $\mathrm{m} / \mathrm{z} 100$ to $\mathrm{m} / \mathrm{z} 1,000$ with a resolving power setting of $60,000 \mathrm{FWHM}$ (at $\mathrm{m} / \mathrm{z} 400$ ). Data were recorded using Qualitative Analysis B.07.00 and PCDL Manager B.07.00 (Agilent). For MS/MS measurements, the energy of collision-induced dissociation $(10 \mathrm{eV} ; 20 \mathrm{eV}$; $40 \mathrm{eV}$ ) was used. The obtained $\mathrm{m} / \mathrm{z}$ values were compared with the molecular weights of standard DON and its probable degraded product.

\section{Results and Discussion}

\section{Morphology of Graphene/ZnO Photocatalysts}


A series of graphene/ $\mathrm{ZnO}$ hybrid photocatalysts have been obtained by a simple hydrothermal treatment of $\mathrm{ZnO}$ nanoparticles and graphene oxide in water, and the typical sample were denoted as GZ0.3. This treatment process under the hydrothermal condition will easily reduce GO to GR, while simultaneously, the particles of $\mathrm{ZnO}$ are dispersed on the graphene sheet. Figure 1 displays the sample digital photographs and electron microscopy images of the final solid product GZ0.3 with 0.3 wt.\% weight addition of graphene. As shown in Figure 1a, the color exhibited grey white for GZ0.3, similar phenomenon is also observed for other weight addition of graphene for GZX series materials. The morphology of the typical products was investigated by SEM and TEM. Figure $1 \mathrm{~b}$ and $1 \mathrm{c}$ show the SEM and TEM images of GZ0.3, from which it is seen that $\mathrm{ZnO}$ nanoparticles were homogeneously and tightly wrapped by graphene sheets without aggregation. The above observation further demonstrated the presence of graphene sheets helped $\mathrm{ZnO}$ nanoparticles from agglomeration during the growth of the graphene/ $\mathrm{ZnO}$ hybrid, and enabled a good wrapped morphology of these oxide particles by graphene sheets.

\section{Enhancement of Photocatalytic Activity}

The photocatalytic activities of the graphene/ZnO hybrid, using photodegradation of DON as model reaction under UV light $(365 \mathrm{~nm})$, are shown in Figure 2a and Figure $2 \mathrm{~b}$. Figure 2a shows the DON photodegradation rate constants on graphene/ZnO hybrid under UV light irradiation. The degradation kinetic data were fitted by using a pseudo first-order equation with a simplified Langmuir-Hinshelwood model to obtain the rate constant $\mathrm{k}^{28}$ The introduction of graphene into $\mathrm{ZnO}$ matrix drastically increased the 
rate constant. The photocatalytic activity trend follows the order: GZ0.3 > GZ10.0 > GZ3.0 > GZ5.0 > GZ0.1 > GZ8.0, while the sample of GZ0.3 shows the highest reaction rate constant of $0.10411 \mathrm{~min}^{-1}$. More graphene leads to a significant decrease of photocatalytic activity. Clearly, when the weight ratio of graphene is $8.0 \mathrm{wt} \%$, the activity of GZ8.0 is even lower than GZ0.1. Although graphene is beneficial for charge separation of the graphene- $\mathrm{ZnO}$ hybrids, it will shade $\mathrm{ZnO}$ at too much addition. Therefore, due to the balance between charge separation and light harvesting, the photocatalytic activity of graphene/ZnO hybrid firstly increases and then decreases with the increasing of graphene addition. As show in Figure 2b, the rapid degradation of DON and intermediate products over the graphene/ZnO hybrid photocatalysts was clearly seen from the change of HPLC spectra of the solution in the course of the degradation. The HPLC chromatograms show increasing peaks of intermediate products at $\mathrm{t}=1.63 \mathrm{~min}, \mathrm{t}=2.71 \mathrm{~min}$ and $\mathrm{t}=2.97 \mathrm{~min}$ with prolonging irradiation time, which the peak of intermediate products at $\mathrm{t}=1.63 \mathrm{~min}$ to $240 \mathrm{~min}$ and $\mathrm{t}=2.97 \mathrm{~min}$ to 15 min was disappeared, but the peak of intermediate products at $\mathrm{t}=2.71$ min was still increased with prolonging irradiation time to $300 \mathrm{~min}$, indicating these three retentiong times correspond to two types $(t=1.63 \mathrm{~min}$ and $\mathrm{t}=2.97 ; \mathrm{t}=2.71 \mathrm{~min})$ of intermediate products. The main peak of DON at $\mathrm{t}=7.32 \sim 7.39$ min was totally disappeared with prolonging irradiation time to $15 \mathrm{~min}$, which means the DON can be quickly photodegraded by graphene/ZnO hybrid GZ0.3 under UV light (365 nm).

The trend of DON and intermediate products can be obviously observed in Figure 3a, the peak of intermediate products at $t=2.71$ min was continuously increased and are 
maintain not decomposed and the other three peaks $(\mathrm{t}=1.63 \mathrm{~min}, \mathrm{t}=2.97 \min , \mathrm{t}=$ 7.32 7.39 $\mathrm{min}$ ) were eventually disappeared, which indicated the structure and property of intermediate products at $\mathrm{t}=2.71 \mathrm{~min}$ could be investigated in further work. Figure $3 \mathrm{~b}$ shows the hybrid led to a significant photodegradation of DON in comparison with pure $\mathrm{ZnO}$, traditional photocatalysts $\mathrm{P} 25, \mathrm{~g}-\mathrm{C}_{3} \mathrm{~N}_{4}$ and photolysis. About $99 \%$ of the added DON (15 ppm) was degraded by graphene/ZnO hybrids within $30 \mathrm{~min}$, which is 3.1 times than pure $\mathrm{ZnO}$.

The way of isocratic and gradient elution for product was performed to study the amount and type of the intermediate and then definite the mechanism for photodegradation for DON further. The result was shown in Figure 4a and Figure 4b, there were three peaks $(\mathrm{t}=2.71 \mathrm{~min}, \mathrm{t}=3.55 \mathrm{~min}, \mathrm{t}=6.97 \mathrm{~min})$ of intermediate products appeared and the peak of intermediate products at $\mathrm{t}=2.71 \mathrm{~min}$ was continuously increased while the other peak $(t=3.55 \mathrm{~min})$ were eventually disappeared however the peak at $\mathrm{t}=6.97 \mathrm{~min}$ was unchanged with prolonging irradiation time to $120 \mathrm{~min}$, the products of gradient elution were consistent with the result. Based on above analysis, the DON molecule may have been degraded to small fragment molecular by ring opening and could be destroyed the fragment structure further with higher excitation energy over graphene/ZnO hybrid photocatalyst.

\section{Structure of Graphene-ZnO Hybrid}

The XRD patterns of the samples are shown in Figure 5a, the broad XRD peak of the freeze-dried SGH indicates the poor ordering of graphene sheets along their stacking direction and reflects that the framework of the SGH is composed of few-layer stacked 
graphene sheets. The interlayer spacing of the freeze-dried SGH was calculated to be $3.76 \AA$. This value is much lower than that of GO precursor $(6.94 \AA)$ while slightly higher than that of natural graphite $(3.36 \AA) .{ }^{29,30}$ These results suggest the existence of $\pi-\pi$ stacking between graphene sheets in the SGH and also the presence of residual oxygenated functional groups on reduced GO sheets. Thanks to these residual hydrophilic oxygenated groups, the reduced GO sheets can encapsulate $\mathrm{ZnO}$ in the process of hydrothermal reduction. This factor together with the $\pi-\pi$ stacking of graphene sheets resulted in the successful construction of the graphene/ZnO hybrids. It is found that the samples ( $\mathrm{ZnO}, \mathrm{GZ0.1}$, GZ3.0) present the typical XRD diffraction character of wurtzite structure according to the standardized JCPDS (36-1451) card. However, no characteristic peaks of graphene are observed for either GZ0.1 or GZ3.0 in the corresponding region, which may arise from the factors that the content of the graphene is low and that the deposited $\mathrm{ZnO}$ particles prevent the graphene sheets from forming the inerratic stacks. The graphene/ZnO hybrid shows the similar broader diffraction peaks to the pure $\mathrm{ZnO}$ as prepared, which indicates that the size of $\mathrm{ZnO}$ particles is smaller. Furthermore, the diffraction peaks of the graphene/ZnO hybrid are unchanged compared with that of pure $\mathrm{ZnO}$, which indicates that the lattice constants of $\mathrm{ZnO}$ have unchanged because of surface hybridization of the carbonaceous material. ${ }^{31}$

The FTIR spectra of graphene and graphene/ZnO hybrids are shown in Figure $5 \mathrm{~b}$. Figure $5 \mathrm{~b}$ presents typical fingerprint groups of GO, including carboxylic species, hydroxyl species and epoxy species $\left(\mathrm{C}=\mathrm{O}, 1734 \mathrm{~cm}^{-1}\right.$; OH deformation, $1400 \mathrm{~cm}^{-1}$; the C-OH stretching, $1230 \mathrm{~cm}^{-1}$; C-O-C (epoxy group) stretching, $1061 \mathrm{~cm}^{-1}$; skeletal ring 
stretch, $\left.1624 \mathrm{~cm}^{-1}\right){ }^{32}$ After hydrothermal reduction, the peak of SGH (self-assembled graphene hydrogel) at $1058 \mathrm{~cm}^{-1}$ corresponding to the $\mathrm{C}-\mathrm{O}-\mathrm{C}$ stretching vibration decreased significantly, and the peaks at $1400 \mathrm{~cm}^{-1}$ attributed to the $\mathrm{OH}$ deformation became relatively small. ${ }^{33}$ The absorption characteristic band clearly shows the skeletal vibration of the graphene sheets, indicating the formation of graphene structure. The hybridization between $\mathrm{ZnO}$ and graphene sheets resulted in some changes in the FTIR spectrum. Compared to graphene, it can be found that many new peaks were introduced into the FTIR spectrum of graphene after hybridization. These new peaks at $1572 \mathrm{~cm}^{-1}$, $1236 \mathrm{~cm}^{-1}$ and $1073 \mathrm{~cm}^{-1}$ were observed from the FTIR spectrum of GZ3.0 and appeared at significant intensities, which indicated that there existed strong chemical interaction between graphene and $\mathrm{ZnO}$ molecules. Furthermore, the peak located at $2164 \mathrm{~cm}^{-1}$ corresponding to characteristic functional group of $\mathrm{ZnO}$ disappeared, which can be ascribed to surface hybridization of graphene that the surface of $\mathrm{ZnO}$ was coated by graphene which covered the vibration signal of $\mathrm{ZnO}$. Therefore, the surface hybrid structure of graphene/ZnO can be confirmed by FTIR spectrum definitely, which play a key role in enhancing the photocatalytic efficiency for DON photo-degradation.

The Raman spectra of pristine GO and graphene/ZnO hybrid are shown in Figure 5c, from which it can be found that the GO (Figure S1) shows the D and G bands at 1348 and $1586 \mathrm{~cm}^{-1}$. The $\mathrm{G}$ band is attributed to all $\mathrm{sp}^{2}$ carbon forms and provides information on the in-plane vibration of $\mathrm{sp}^{2}$ bonded carbon atoms while the $\mathrm{D}$ band suggests the presence of $\mathrm{sp}^{3}$ defects. ${ }^{31}$ Compared with graphene, the D band was slightly red-shifted by $6 \mathrm{~cm}^{-1}$ of the nanocomposites while the $\mathrm{G}$ band showed a 
blue-shift of $2 \mathrm{~cm}^{-1}$. These shifts in the Raman peak could be attributed to the chemical interaction between $\mathrm{ZnO}$ and graphene. Raman spectroscopy is also utilized to investigate the single-, bi-, and multilayer characteristics of graphene and graphene oxide layers. ${ }^{34}$ For instance, it was shown that the $\mathrm{G}$ band of the single-layer graphene, located at $1592 \mathrm{~cm}^{-1}$, shifts about $2 \mathrm{~cm}^{-1}$ into higher wavenumbers indicated more contribution of single-layer graphene sheets in the graphene/ZnO hybrid sample. In general, the $\mathrm{I}_{\mathrm{D}} / \mathrm{I}_{\mathrm{G}}$ intensity ratio is a measure of disorder degree and average size of the $\mathrm{sp}^{2}$ domains in graphite materials. ${ }^{35-37}$ Hence, a higher $\mathrm{I}_{\mathrm{D}} / \mathrm{I}_{\mathrm{G}}$ peak intensity ratio indicate more defects and disorders of the graphitized structures containing the disorders caused on the surface of the carbon platelets. Compared with graphene $\left(\mathrm{I}_{\mathrm{D}} / \mathrm{I}_{\mathrm{G}}=0.92\right)$, the increased $\mathrm{I}_{\mathrm{D}} / \mathrm{I}_{\mathrm{G}}$ intensity ratio for $\mathrm{GZ3.0}\left(\mathrm{I}_{\mathrm{D}} / \mathrm{I}_{\mathrm{G}}=1.02\right)$ is observed, implies a decrease in the size of the in-plane $\mathrm{sp}^{2}$ domains and formation of the defects and disorders on the surface of graphene. Above results are consistent with the results in FTIR characterization, revealing the restablishment of the conjugated graphene network $\left(\mathrm{sp}^{2}\right.$ carbon).

The absorption range of light plays an important role in the photocatalysis, Figure $5 \mathrm{~d}$ shows the UV-vis diffuse reflectance spectroscopy (DRS) of samples, there is not too obvious red shift of ca. 20 30 $\mathrm{nm}$ in the absorption edge of GZ3.0 powder, compared to pristine $\mathrm{ZnO}$. The change in the reflectance spectra at approximately $2.95 \mathrm{eV}(419 \mathrm{~nm})$ suggests that the energy band gap of the GZ3.0 is simply associated with broad contribution from absorbance of graphene. In addition, the introduction of graphene on $\mathrm{ZnO}$ surface induces the increased light absorption intensity in the visible region. The 
stronger absorption intensity in $\mathrm{UV}$ and visible regions for GZ3.0 than $\mathrm{ZnO}$ is a key factor for higher photocatalytic activity. It can also be clearly observed that $\mathrm{ZnO}$ with graphene coating with disorders structure showed enhanced intensity of light absorption.

TGA is an effective analytical technique to evaluate the content of the graphene in the hybrid of graphene/ZnO photocatalysts. ${ }^{31}$ Figure $6 a$ shows the curves of thermal gravimetric analysis and differential thermal analysis in air atmosphere for the graphene and graphene/ZnO hybrid. The graphene oxide powders exhibited three obvious steps of mass loss: the evaporation loss of adsorbed water about at $100{ }^{\circ} \mathrm{C}$ (about $5 \%$ of weight loss), the removal of oxygen-containing groups accompanied by the liberation of $\mathrm{C}_{\mathrm{x}} \mathrm{H}_{\mathrm{y}} \mathrm{O}_{\mathrm{z}}$ species (about $15 \%$ of weight loss), as shown in Figure 6b, corresponding to an obvious exothermic differential scanning calorimetry (DSC) peak at $203{ }^{\circ} \mathrm{C}$, and the combustion of carbon layers for graphene with a strong and sharp exothermic peak at $500{ }^{\circ} \mathrm{C}$ in DSC curve. The SGH shows only one obvious steps of mass loss that has no peak of water for physical adsorption in comparison with GO, which is located at about $573{ }^{\circ} \mathrm{C}$ due to the removal of oxygen-containing groups. However, the curve for the graphene/ZnO hybrid sample (GZ3.0) did not exhibit three such clear steps of mass loss and had a later onset of weight loss (exclude the mass loss of adsorbed water) than GO, which showed two weak exothermic peaks at $150^{\circ} \mathrm{C}$ and $350{ }^{\circ} \mathrm{C}$, respectively. The TGA curve of GZ3.0 sample shows a dramatical mass loss at $150{ }^{\circ} \mathrm{C}$, which is a lower decomposition temperature for oxygen group compared with $\mathrm{GO}$, indicating that the thermal stability of GO is decreased after hybrided with $\mathrm{ZnO}$ 
(inserted graph in Figure 6a). The curve of pure $\mathrm{ZnO}$ shows nearly no mass loss at 900 ${ }^{\circ} \mathrm{C}$ because of dry and high purity for $\mathrm{ZnO}$ sample which with no adsorbed water and acetate group (Figure 6b). Based on the above analysis, these results suggested that the GZ3.0 was stabilized by the coated graphene on the surface of $\mathrm{ZnO}$ because of the strong chemical interaction between graphene and $\mathrm{ZnO}$ particles, leading to delayed weight loss of graphene. According to the TGA curve, the weight content of graphene in the composite GZ3.0 was roughly evaluated to be $2.6 \%$, which is close to the theoretical dosage of GO (3\%) while there may has a few small losses that could attributed the effect in the process of hydrothermal reduction.

$\mathrm{N}_{2}$ adsorption desorption measurements with Brunauer-Emmett-Teller (BET) method was used to determine the surface area of the composites and to check the possibility of interconnected pores in the nanomaterials. As shown in Figure 7, the nonlimiting adsorption at high $\mathrm{P} / \mathrm{P}_{0}$ for graphene/ZnO hybrid samples is characteristic of a Type IV loop, and the overall shapes of the three samples indicated their meso- and macroporous characteristics. The BET data was summarized in Table 1, the result analysis revealed that the graphene/ZnO hybrid had a surface area of $58.9 \mathrm{~m}^{2} \mathrm{~g}^{-1}$, being much higher than $10.4 \mathrm{~m}^{2} \mathrm{~g}^{-1}$ of the pure $\mathrm{ZnO}$ indicating that graphene is an excellent surface hybrid material for the immobilization of $\mathrm{ZnO}$. The measured specific surface area $\left(388.9 \mathrm{~m}^{2} \mathrm{~g}^{-1}\right)$ and water absorption ability (96.4\%) of SGH was much higher than GZ0.3 for $58.9 \mathrm{~m}^{2} \mathrm{~g}^{-1}$ and $85.2 \%$, respectively. In addition, the specific surface area of SGH was much less than theoretical value of an monolayer graphene sheet $\left(2630 \mathrm{~m}^{2}\right.$ $\left.\mathrm{g}^{-1}\right),{ }^{39}$ possibly because a large amount of polar groups such as hydroxyl and carboxyl 
on the graphene and the strong hydrogen bonding caused tight sheet associations. Thanks to these residual hydrophilic oxygenated groups, the reduced monolayer GO sheets can encapsulate $\mathrm{ZnO}$ in the process of hydrothermal reduction. ${ }^{29}$ We calculated the pore-size distribution by using the Barrett-Joyner-Halenda (BJH) method from the desorption branch of the isotherm. In comparison with $\mathrm{ZnO}$ samples, the graphene/ZnO hybrid involved much more mesopores with the pore size centered at $3.8 \mathrm{~nm}$ and the average pore size located at $9.0 \mathrm{~nm}$ (the inset of Figure 7), the pores are uniformly distributed over GZ0.3 sample. The $\mathrm{dV} / \mathrm{dD}$ of GZ0.3 is about $0.01 \mathrm{~cm}^{3} / \mathrm{g}$, while is about $0.25 \mathrm{~cm}^{3} / \mathrm{g}$ for $\mathrm{SGH}$, the slight decrease of $\mathrm{dV} / \mathrm{dD}$ may be ascribed to monolayer graphene sheet tightening the stacking structure of the $\mathrm{ZnO}$ powder.

\section{Mechanism on Enhancement of Photocatalytic Activity and Probable Degradation Products of DON}

To reveal the photocatalytic mechanism further, Figure 8 shows the photodegradation of DON with the addition of hydroxyl radical scavenger $(t \mathrm{BuOH})^{40}$ and hole scavenger (EDTA-2Na) ${ }^{41}$ under UV light irradiation. As shown in Figure 8, the photocatalytic activity of GZ0.3 decreases largely by the addition of hole scavengers (EDTA-2Na), while reduces slightly with the addition of hydroxyl radical scavengers, indicating that the hydroxyl radical are not the main oxidative species for GZ0.3 samples. That is, hole mainly plays more key role than hydroxyl radical in the UV light photocatalytic process. In addition, $\mathrm{N}_{2}$ is also a good detective molecular to make certain the effect of $\mathrm{O}_{2}$, shown in Figure 8. Under the anoxic suspension, the photodegraded rate of DON in GZ0.3 system is largely prohibited, indicating $\mathrm{O}_{2}$ is even 
more important in the photodegradation process that produces more superoxide radicals $\left({ }^{-} \mathrm{O}_{2}\right)$ in comparison with the addition of $t \mathrm{BuOH}$ and EDTA-2Na system in graphene/ZnO hybrid system. Base on the above analysis, it can be concluded that the photooxidation mechanism occurring on the surface of graphene/ZnO hybrid may involve the direct oxidizing reaction of DON with superoxide radicals $\left(\bullet^{-} \mathrm{O}_{2}\right)$ and holes. To confirm the degradation product further, the degradation products of DON under UV light were investigated and performed by ESI/MS analysis. The HPLC peak at 6.70 min of total ion chromatogram (TIC) and MS spectra in the positive ESI mode for DON are shown in Figure 9a and Figure 9b, which exhibits obvious enhanced photocatalytic activity for DON and appears three peaks of intermediate products under UV light irradiation. ESI/MS analysis confirmed the decreasing trend of DON and the degradation product which the secondary mass spectrogram $\left(\mathrm{MS}^{2}\right)$ in the positive ESI mode are depicted in Figure 10a and Figure 10b, the dominant molecular ion peak at $\mathrm{m} / \mathrm{z} 281.8747$ which is similar to that of DOM-1. Structurally, DON is a polar organic compound that belongs to the type B trichothecenes and the molecule contains three free hydroxyl groups $(-\mathrm{OH})$, which are associated with its toxicity. ${ }^{42}$ De-epoxy DON (DOM-1) is the primary metabolite produced by reduction of the 12,13 -epoxygroup to a diene, which eliminated toxicity of trichothecenes by selective cleavage of their toxic 12,13-epoxy group and transformed DON into the nontoxic, de-epoxide of DON. The adduct ion for DON was found at $\mathrm{m} / \mathrm{z}=333.0932$, which refers to DON combines with two molecules of water to form adduct $\left[\mathrm{M}+\mathrm{H}^{+}+2 \mathrm{H}_{2} \mathrm{O}\right]^{+}$and this adduct ion of DON at 333.0932 has also been reported by Razzazi-Fazeli et al. ${ }^{13,43}$ The other ion for DON was 
found at $\mathrm{m} / \mathrm{z}=298.8996$, which may be attributed to the photo-reduction product $[\mathrm{M}+$ $\mathrm{H}^{+}{ }^{+}$of DON under UV light irradiation over graphene/ZnO hybrid. These results indicate that DON could be photodegraded rapidly in aqueous solution under UV light irradiation and may form three new degradation products. In a word, the TIC and MS ${ }^{2}$ analysis for DON confirmed the degraded product formation and gave $\mathrm{m} / \mathrm{z}$ values of 281.8747, 333.0932 and 298.8996 in positive-ion mode, suggesting that DON was reduced by photo-degradation and the new product which deduced is DOM-1, $\left[\mathrm{M}+\mathrm{H}^{+}\right.$ $\left.+2 \mathrm{H}_{2} \mathrm{O}\right]^{+},[\mathrm{M}+\mathrm{H}]^{+}$, respectively.

On the basis of the above analysis, as shown in Scheme 1, it can be concluded that the enhancement of the photocatalytic activity of the graphene/ZnO hybrid for DON may be mainly attributed graphene which has superior electrical conductivity, enhance adsorption ability and a good hybrid layer which serve as adsorption active sites. Then a possible mechanism for the synergetic and competitive photocatalysis is proposed. As shown in Scheme 1a, it has been observed that the initial introduction of graphene into $\mathrm{ZnO}$ matrix drastically increased the rate constant which mainly attributed to the synergetic effect of graphene and $\mathrm{ZnO}$. It was reported that graphene as electron acceptor was a competitive candidate for the electron acceptor material due to its two dimensional p-conjugation structure. We may consider that in the graphene/ZnO hybrid, the excited electrons of $\mathrm{ZnO}$ can quickly transfer from the conduction band of $\mathrm{ZnO}$ to the graphene, and then effectively suppresses the recombination of photogenerated charge carriers, leaving more charge carriers to form highly reactive species (superoxide radicals $\left(\cdot{ }^{-} \mathrm{O}_{2}\right)$ and holes) and promote the degradation of DON molecules, 
the similar result have been confirmed by Zhang et al. ${ }^{20}$ It is known that graphene has a high work function, which enables an easy and fast transfer of conduct band electron of $\mathrm{ZnO}$, being similar to that of gold. ${ }^{44,45}$ The photogenerated electrons have longer lifetime and higher efficiency to reduce electron acceptor once it is transferred from conduction band of $\mathrm{ZnO}$ to graphene and then form the superoxide radicals $\left(\cdot{ }^{-} \mathrm{O}_{2}\right)$. As shown in Scheme $1 \mathrm{~b}$, the adsorption ability of graphene/ $\mathrm{ZnO}$ hybrid is superior to photocatalytic ability while the weight ratio of graphene exceed $8.0 \mathrm{wt} \%$ which can probably be ascribed to the increase of specific surface as well as the adsorptive site on the graphene/ZnO hybrid. Although graphene is beneficial for charge separation of the graphene/ZnO hybrid, it will shade $\mathrm{ZnO}$ at too much addition while the competitive effect of adsorption and photocatalysis exceeds synergetic interaction between graphene and $\mathrm{ZnO}$. The synergetic interaction means graphene provide a good adsorption site and support substrate for the deposition of DON molecules which making the graphene/ $\mathrm{ZnO}$ hybrid have a two-dimensional nanostructure while the competitive effect implies the photocatalytic effect is weaken along with the enhancement of adsorption which could also lead to increment of removal rate for DON. Therefore, due to the balance between charge separation and light harvesting, the photocatalytic activity of graphene/ZnO hybrid firstly increases and then decreases with the increasing of graphene addition. It is concluded that graphene exhibits advantages of adsorptive ability and $\mathrm{ZnO}$ shows enhancement of photocatalytic performance by graphene hybridization. The DON molecules are largely adsorbed onto graphene surface and then photodegraded by graphene/ZnO hybrid synergistically or 
competitively under UV irradiation. The graphene/ZnO hybrid can be considered an ideal and effective material to remove DON molecules in aqueous solution due to the synergetic or competitive effect of adsorption and photocatalysis.

\section{Conclusions}

In conclusion, $\mathrm{ZnO} /$ graphene monolayer hybrid with high photocatalytic performance has been successfully and directly prepared via a simple one-step hydrothermal method. The hybrid photocatalysts possess enhanced specific surface area, extended photoresponding range and intensive light intensity, which contribute to the superior photocatalytic activity for the photodegradation of DON under the irradiation of UV light. The UV light photocatalytic activity of $\mathrm{ZnO}$ /graphene hybrid GZ0.3 was 3.1 times than pure $\mathrm{ZnO}$ and about $99 \%$ of DON (15 ppm) could be photodegraded within 30 min totally while there were three peaks of intermediate products appeared. The ESI/MS analysis confirmed the photodegradation of DON and investigated the degradation product with the secondary mass spectrogram in the positive ESI mode, which gave $\mathrm{m} / \mathrm{z}$ values of $281.8747,333.0932$ and 298.8996, suggesting that DON was reduced by photodegradation and the new product formed is DOM-1, $\left[\mathrm{M}+\mathrm{H}^{+}+2 \mathrm{H}_{2} \mathrm{O}\right]^{+},[\mathrm{M}+\mathrm{H}]^{+}$, respectively. The superior photocatalytic activity of the hybrid could be attributed to the direct oxidizing reaction of DON with superoxide radicals $\left({ }^{\bullet} \mathrm{O}_{2}\right)$ and holes. The graphene/ZnO hybrid can be considered an ideal and effective material to remove DON molecules in aqueous solution due to the synergetic or competitive effect of adsorption and photocatalysis. This work can provide important inspirations for developing of graphene-based photocatalysts for 
mycotoxin detoxification and environmental remedial applications.

\section{Supporting Information}

Supplementary data (Raman spectra, and synthesis details of graphene oxide) associated with this article can be found in the online version. This information is available free of charge via the Internet at http://pubs.acs.org/.

\section{Acknowledgment}

This work was partly supported by Chinese National Science Foundation (21607034), National Basic Research Program of China (973 Program (2013CB127805), The National Key Technology R\&D Program of China (2015BAK43B00) and Special Fund for Grain-scientific Research in the Public Interest in China (201513006). 


\section{Notes and references}

(1) Petska J. J. Deoxynivalenol: toxicity, mechanisms and animal health risks. Anim Feed Sci Technol. 2007,137, 283-298.

(2) Numanoglu, E.; Gokmen, V.; Uygun, U.; Koksel, H. Thermal degradation of deoxynivalenol during maize bread baking. Food Additives and Contaminants. 2012, 29, 423-430.

(3) Bretz, M.; Beyer, M.; Cramer, B.; Knecht, A.; Humpf, H. U. Thermal Degradation of the Fusarium Mycotoxin Deoxynivalenol. J. Agric. Food Chem. 2006, 54, 6445-6451.

(4) Alassane-Kpembi, I.; Kolf-Clauw, M.; Gauthier, T.; Abrami, R.; Abiola, F. A.; Oswald, I. P.; Puel, O. New insights into mycotoxin mixtures: the toxicity of low doses of type B trichothecenes on intestinal epithelial cells is synergistic. Toxicol. Appl. Pharmacol. 2013, 272, 191-198.

(5) Kluger, B.; Bueschl, C.; Lemmens, M.; Berthiller, F.; Haubl, G.; Jaunecker, G.; Adam, G.; Krska, R.; Schuhmacher, R. Stable isotopic labelling-assisted untargeted metabolic profiling reveals novel conjugates of the mycotoxin deoxynivalenol in wheat. Anal. Bioanal. Chem. 2013, 405, 5031-5036.

(6) Stanic, A.; Uhlig, S.; Solhaug, A.; Rise, F.; Wilkins, A. L.; Miles. C. O. Nucleophilic Addition of Thiols to Deoxynivalenol. J. Agric. Food Chem. 2015, 63(34), 7556-7566.

(7) Park, B. J.; Takatori, K.; Sugita-Konishi, Y.; Kim, I. H.; Lee, M. H.; Han, D. W.; 
Chung, K. H.; Hyun, S. O.; Park, J. C. Degradation of mycotoxins using microwave-induced argon plasma at atmospheric pressure. Surface \& Coatings Technology. 2007, 201, 5733-5737.

(8) Van-Dyck, P. J.; Tobback, P.; Feys, M.; Voorde, H. V. Sensitivity of aflatoxin B1 to ionizing radiation. Appl. Environ. Microbiol. 1982, 43, 1317-1319.

(9) Hooshmand, H.; Klopfenstein, C. F. Effects of gamma irradiation on mycotoxin disappearance and amino acid contents of corn, wheat, and soybeans with different moisture contents. Plant Foods Hum. Nutr. 1995, 47, 227-238.

(10) Youssef, B. M.; Mahrous, S. R.; Aziz, N. H. Effect of gamma irradiation on aflatoxin B1 production by Aspergillus flavus in ground beef stored at $5{ }^{\circ} \mathrm{C}$. J. Food Safety. 1999, 19, 231-239.

(11)Xie, F.; Ha, Y. M.; Wang, F. Studies on Gamma irradiation induced degradation of chloramphenicol in aqueous solution, Chin. J. Radiat. Res. Radiat. Process. 2008, $26,151-156$.

(12)Wang, F.; Xie, F.; Xue, X. F.; Wang, Z. D.; Fan, B.; Ha, Y. M. Structure elucidation and toxicity analyses of the radiolytic products of aflatoxin B1 in methanol-water solution. Journal of Hazardous Materials. 2011, 192, 1192-1202.

(13)Mishra, S.; Dixit, S.; Dwivedi, P. D.; Pandey, H. P.; Das, M. Influence of temperature and $\mathrm{pH}$ on the degradation of deoxynivalenol (DON) in aqueous medium: comparative cytotoxicity of DON and degraded product. Food Additives \& Contaminants: Part A. 2014, 31, 121-131.

(14)Wu, Q. H.; Lohrey, L.; Cramer, B.; Yuan, Z. H.; Humpf, H. U. Impact of 
Physicochemical Parameters on the Decomposition of Deoxynivalenol during Extrusion Cooking of Wheat Grits. J. Agric. Food Chem. 2011, 59, 12480-12485.

(15)Mishra, H. N.; Das, C. A review on biological control and metabolism of aflatoxin. Crit Rev Food Sci Nutr. 2003, 43, 245-264.

(16) Young, J. C.; Zhou, T.; Yu, H.; Zhu, H.; Gong, J. Degradation of trichothecene mycotoxins by chicken intestinal microbes. Food Chem Toxicol. 2007, 45, 136-143.

(17)Bhatkhande, D. S.; Pangarkar, V. G.; Beenackers, A. A. Photocatalytic degradation for environmental applications-a review. J Chem Technol Biotechnol. 2001, 77, 102-116.

(18) Xu, T. G.; Zhang, L. W.; Cheng, H. Y.; Zhu, Y. F. Significantly Enhanced Photocatalytic Performance of $\mathrm{ZnO}$ via Graphene Hybridization and the Mechanism Study. Applied Catalysis B: Environmental. 2011, 101, 382-387.

(19)Berger, C.; Song, Z.; Li, X.; Wu, X.; Brown, N.; Naud, C.; Mayou, D.; Li, T.; Hass, J.; Marchenkov, A. N.; Conrad, E. H.; First, P. N.; Heer, W. A. Electronic Confinement and Coherence in Patterned Epitaxial Graphene. Science. 2006, 312, 1191-1196.

(20)Zhang, H.; Lv, X. J.; Li, Y. M.; Wang, Y.; Li, J. H. P25-Graphene Composite as a High Performance Photocatalyst. ACS Nano, 2010, 4 (1), 380-386.

(21)Wu, J. L.; Shen, X. P.; Jiang, L.; Wang, K.; Chen. K. M. Solvothermal synthesis and characterization of sandwich-like graphene/ZnO nanocomposites. Appl. Surf. Sci. 2010, 256, 2826-2830.

(22)Zhang, X. Y.; Li, H. P.; Cui, X. L.; Lin, Y. H. Graphene/TiO 2 nanocomposites: 
synthesis, characterization and application in hydrogen evolution from water photocatalytic splitting. J. Mater. Chem. 2010, 20, 2801-2806.

(23)Williams, G.; Kamat, P. V. Graphene-Semiconductor Nanocomposites: Excited-State Interactions between $\mathrm{ZnO}$ Nanoparticles and Graphene Oxide. Langmuir. 2009, 25(24), 13869-13873.

(24)Lambert, T. N.; Chavez, C. A.; Hernandez-Sanchez, B.; Lu, P.; Bell, N. S.; Ambrosini, A.; Friedman, T.; Boyle, T. J.; Wheeler, D. R.; Huber, D. L. Synthesis and Characterization of Titania-Graphene Nanocomposites. J. Phys. Chem. C. 2009, 113(46), 19812-19823.

(25)Gouvea, K.; Wypych, F.; Moraes, S. G.; Duran, N.; Nagata, N.; Peralta-Zamora, P. Semiconductor-assisted photocatalytic degradation of reactive dyes in aqueous solution. Chemosphere. 2000, 40, 433-440.

(26)Dindar, B.; Icli, S. Unusual photoreactivity of zinc oxide irradiated by concentrated sunlight. J. Photochem. Photobiol. A: Chem. 2001, 140, 263-268.

(27)Hummers, W. S., Offeman, R. E. Preparation of Graphitic Oxide. J. Am. Chem. Soc. 1958, 80, 1339-1339.

(28)Li, Y. J.; Li, X. D.; Li, J. W.; Yin, J. Photocatalytic degradation of methyl orange by $\mathrm{TiO}_{2}$-coated activated carbon and kinetic study.Water Res. 2006, 40, 1119-1126.

(29)Xu, Y. X.; Sheng, K. X.; Li, C.; Shi, G. Q. Self-Assembled Graphene Hydrogel via a One-Step Hydrothermal Process. ACS Nano. 2010, 4, 4324-4330.

(30)Murugan, A. V.; Muraliganth, T.; Manthiram, A. Rapid, Facile Microwave-Solvothermal Synthesis of Graphene Nanosheets and Their Polyaniline 
Nanocomposites for Energy Storage. Chem. Mater. 2009, 21, 5004-5006.

(31)Fu, D. Y.; Han, G. Y.; Chang, Y. Z.; Dong, J. H. The synthesis and properties of $\mathrm{ZnO}$-graphene nano hybrid for photodegradation of organic pollutant in water. Materials Chemistry and Physics. 2012, 132, 673-681.

(32)Xu, J.; Wang, L.; Zhu, Y. F. Decontamination of Bisphenol A from Aqueous Solution by Graphene Adsorption. Langmuir. 2012, 28, 8418 -8425.

(33)Chandra, V.; Park, J.; Chun, Y.; Lee, J. W.; Hwang, I. C.; Kim, K. S. Water-dispersible magnetite-reduced graphene oxide composites for arsenic removal. ACS Nano. 2010, 4, 3979-3986.

(34) Yang, D. X.; Velamakanni, A.; Bozoklu, G.; Park, S.; Stoller, M.; Piner, R. D.; Stankovich, S.; Jung, I.; Field, D. A.; Ventrice, C. A.; Ruoff, R. S. Carbon. 2009, 47, $145-152$.

(35) Akhavan, O. Graphene Nanomesh by ZnO Nanorod Photocatalysts. ACS Nano. 2010, 4, 4174-4180.

(36)Gomez-Navarro, C.; Weitz, R. T.; Bittner, A. M.; Scolari, M.; Mews, A.; Burghard, M.; Kern, K. Electronic Transport Properties of Individual Chemically Reduced Graphene Oxide Sheets. Nano Lett. 2007, 7, 3499-3503.

(37)Bai, X. J.; Li Wang, L.; Zhu, Y. F. Visible Photocatalytic Activity Enhancement of $\mathrm{ZnWO}_{4}$ by Graphene Hybridization. ACS Catalysis. 2012, 2, 2769-2778.

(38)Bai, X. J.; Wang, L.; Zong, R. L.; Lv, Y. H.; Sun, Y. Q.; Zhu, Y. F. Performance Enhancement of ZnO Photocatalyst via Synergic Effect of Surface Oxygen Defect and Graphene Hybridization. Langmuir. 2013, 29, 3097-3105. 
(39)Zhu, Y.; Murali, S.; Cai, W.; Li, X.; Suk, J. W.; Potts, J. R.; Ruoff, R. S. Graphene and graphene oxide: synthesis, properties, and applications, $A d v$. Mater. 2010, 22 , 3906-3924.

(40)Hyunjoo, L.; Wonyong, C. Photocatalytic Oxidation of Arsenite in $\mathrm{TiO}_{2}$ Suspension: Kinetics and Mechanisms. Environ. Sci. Technol. 2002, 36, $3872-3878$.

(41)Zhou, J. H.; Deng, C. Y.; Si, S. H.; Shi, Y.; Zhao, X. L. Study on the Effect of EDTA on the Photocatalytic Reduction of Mercury onto Nanocrystalline Titania using Quartz Crystal Microbalance and Differential Pulse Voltammetry. Electrochimica Acta. 2011, 56, 2062-2067.

(42)Nagy, C.M.; Fejer, S. N.; Berek, L.; Molnar, J.; Viskolcz, B. Hydrogen bondings in deoxynivalenol (DON) conformations-a density functional study. J Mol. Struct. 2005, 726, 55-59.

(43)Razzazi-Fazeli, E.; Böhm, J.; Luf, W. Determination of nivalenol and deoxynivalenol in wheat using liquid chromatography-mass spectrometry with negative ion atmospheric pressure chemical ionisation. J Chroma A. 1999, 854, 45-55.

(44) Yu, Y.; Zhao, Y.; Ryu, S.; Brus, L. E.; Kim, K. S.; Kim, P. Tuning the graphene work function by electric field effect. Nano Lett. 2009, 9(10), 3430-3434.

(45)Jiang, G. D.; Lin, Z. F.; Chen, C.; Zhu, L. H.; Chang, Q.; Wang, N.; Wei, W.; Tang, H. Q. $\mathrm{TiO}_{2}$ nanoparticles assembled on graphene oxide nanosheets with high 
photocatalytic activity for removal of pollutants. Carbon, 2011, 49, 2693-2701.

\section{Figure Captions}

Figure 1. Characterization of graphene-ZnO hybrid photocatalysts with $0.3 \mathrm{wt} . \%$ mass ratio of graphene: (a) digital photograph of GZ0.3; (b) SEM image of GZ0.3; (c) TEM image of GZ0.3. 
Figure 2. (a) Apparent rate constants/curves for the photocatalytic degradation of DON over graphene-photocatalyst nanocomposites GZX series. (b) HPLC chromatograms of extension of a time about 300 min over GZ0.3 under the $365 \mathrm{~nm}$ irradiation.

Figure 3. Degradation rate of intermediate product over graphene/ZnO hybrid GZ0.3 under $365 \mathrm{~nm}$ and apparent rate constants of different photocatalysts under $254 \mathrm{~nm}$ for the photocatalytic degradation of DON.

Figure 4. Isocratic elution (a) and gradient elute (b) procedures of HPLC chromatograms for the photocatalytic degradation of $\mathrm{DON}$ over graphene/ZnO nanocomposites GZ0.3 under the $365 \mathrm{~nm}$ irradiation.

Figure 5. Characterization of GZX series photocatalysts and blank: (a) XRD patterns;

(b) FTIR spectra; (c) Raman spectrum; (d) DRS spectra.

Figure 6. (a) TGA and (b) DSC curves of graphene/ZnO photocatalysts and blank controls.

Figure 7. $\mathrm{N}_{2}$ adsorption-desorption isotherm and $\mathrm{BJH}$ pore size distribution of the graphene/ZnO hybrid photocatalyst.

Figure 8. Photodegradation rate curves of DON over GZ0.3 with the additon of hole, radical scavenger and $\mathrm{N}_{2}$ under the irradiation of UV light $(\lambda=365 \mathrm{~nm})$.

Figure 9. Total ion chromatogram (TIC) and MS spectra in the positive ESI mode of DON over GZ0.3 photocatalyst. (a)TIC spectra; (b) MS spectra (Collision energy $=20$ $\mathrm{eV})$.

Figure 10. The MS/MS spectra in the positive ESI mode of the degradation product for DON over GZ0.3 photocatalyst.(a) $\mathrm{M} / \mathrm{Z}=281.8747$; (b) $\mathrm{M} / \mathrm{Z}=333.0932 \quad$ (Collision 
energy $=20 \mathrm{eV})$.

Figure 1 

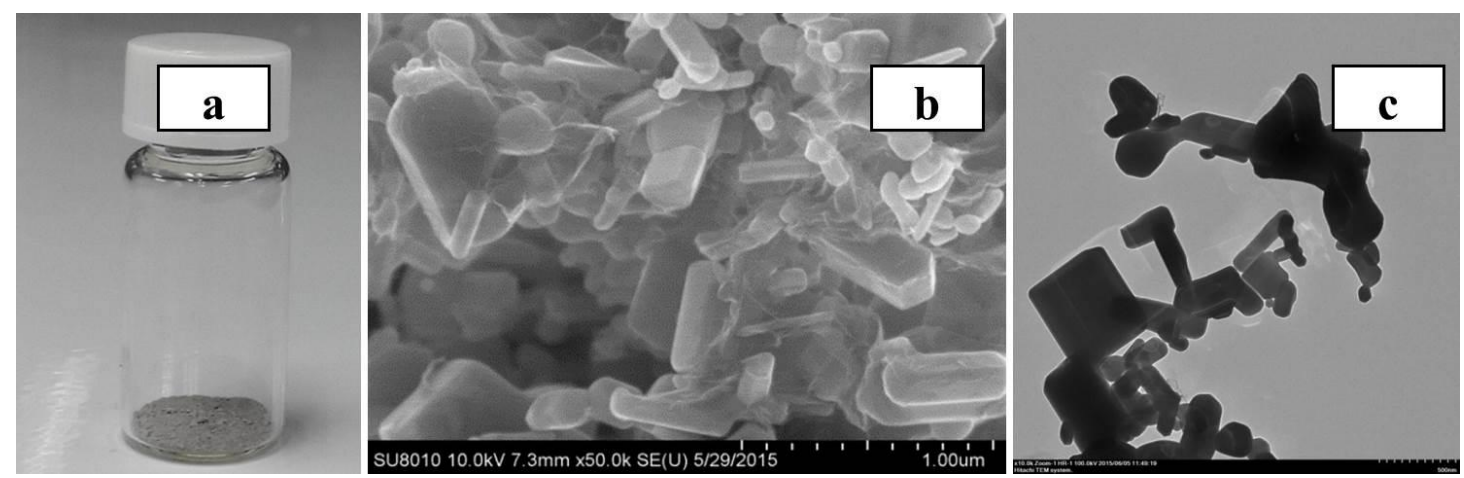

Figure 2
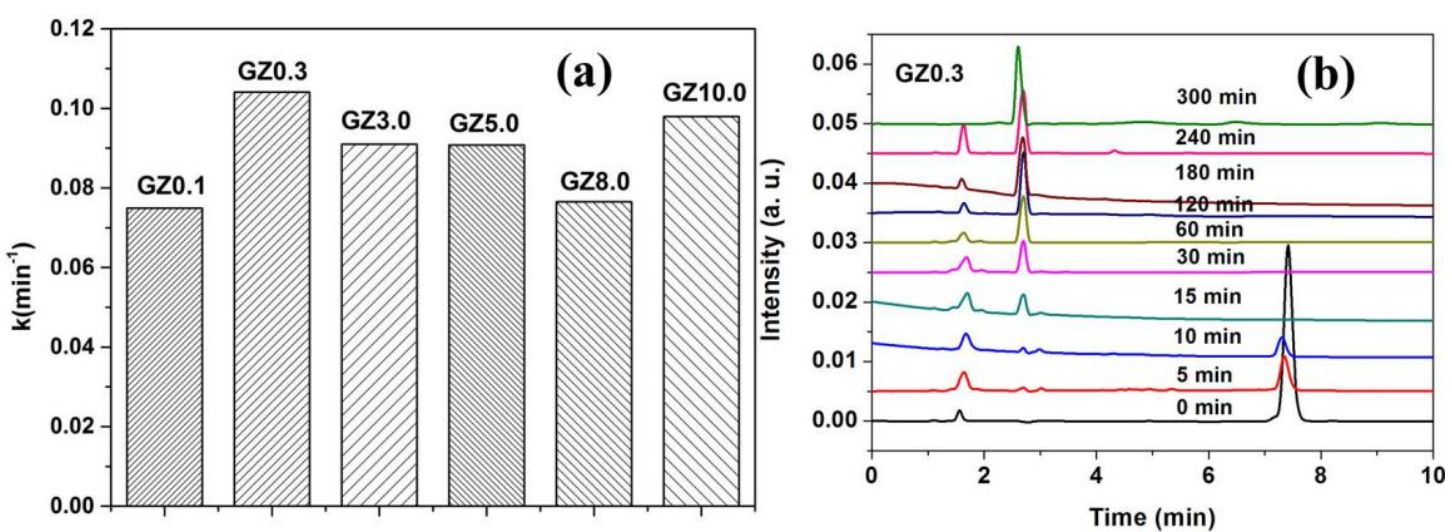

Figure 3

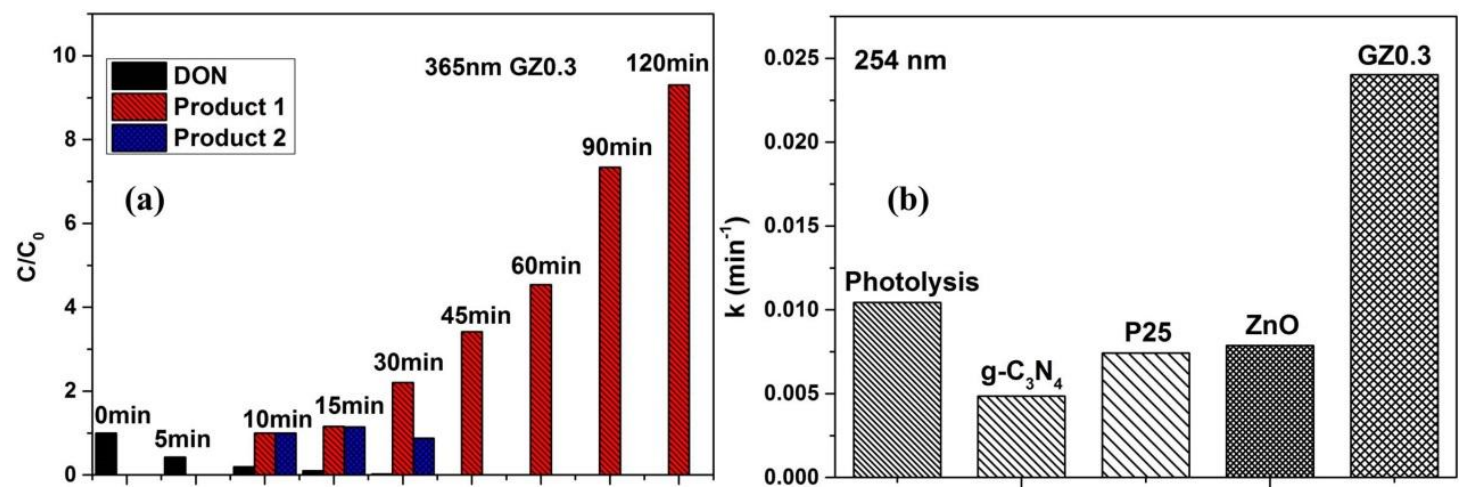

Figure 4 

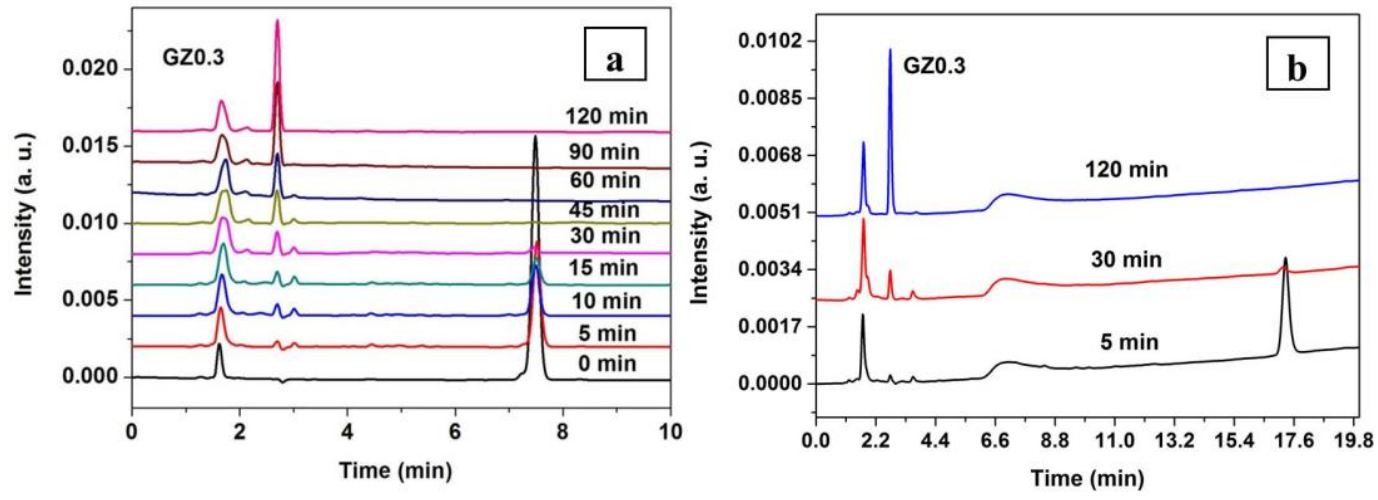

Figure 5
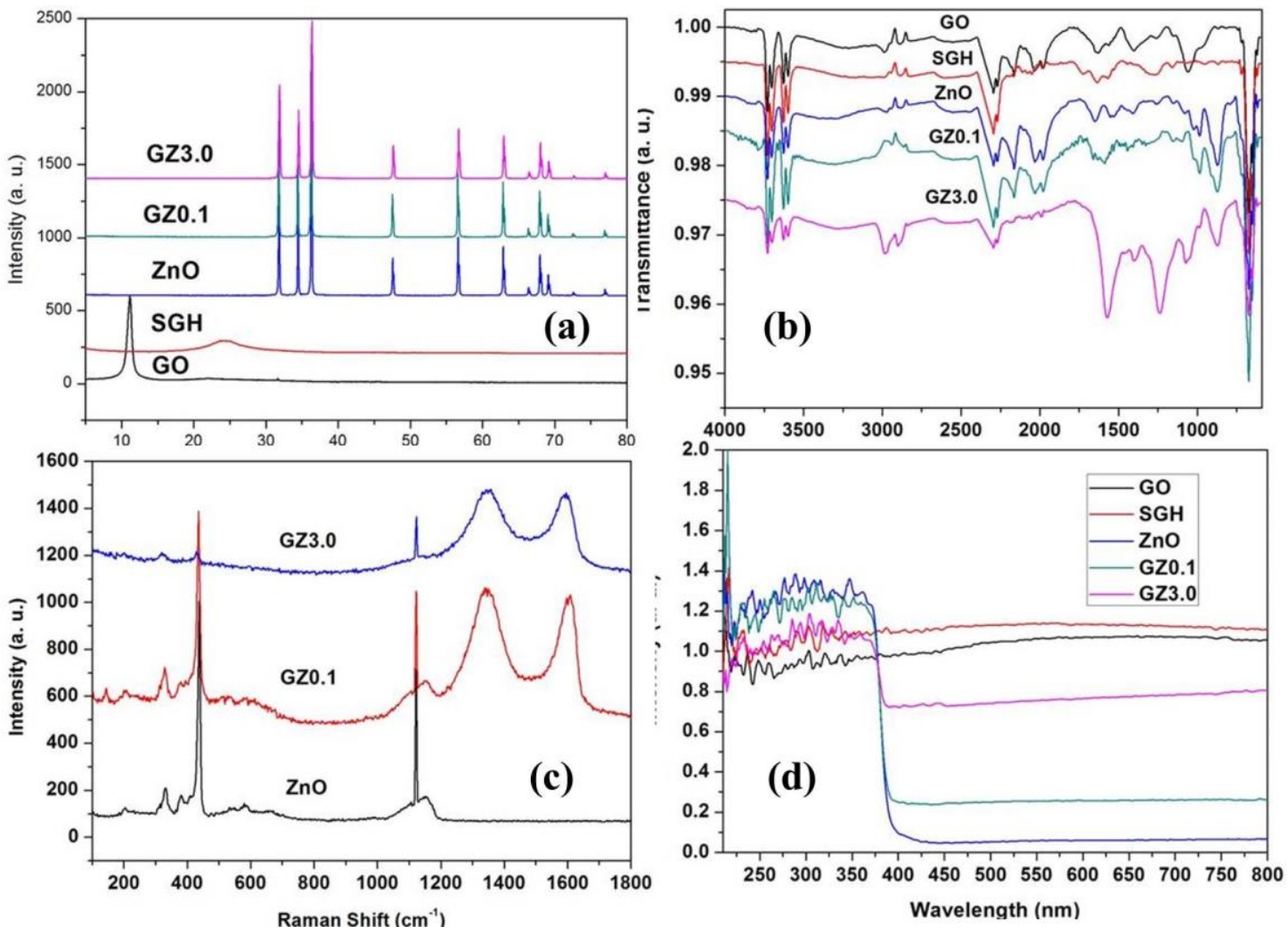

Figure 6 

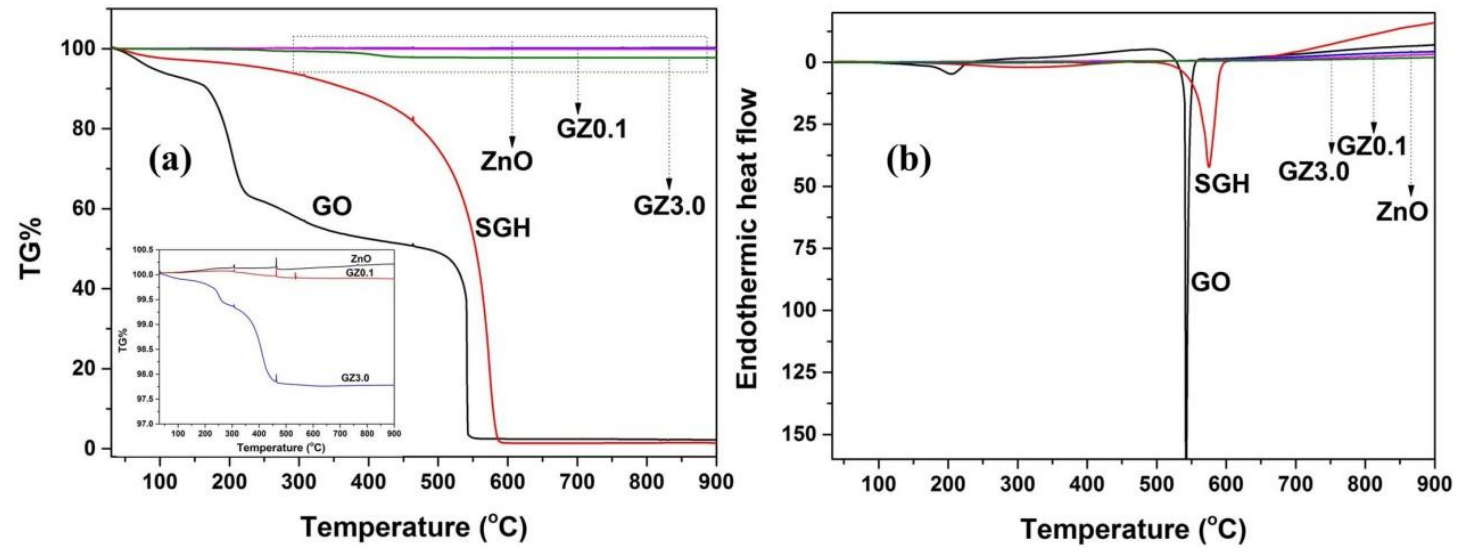

Figure 7 


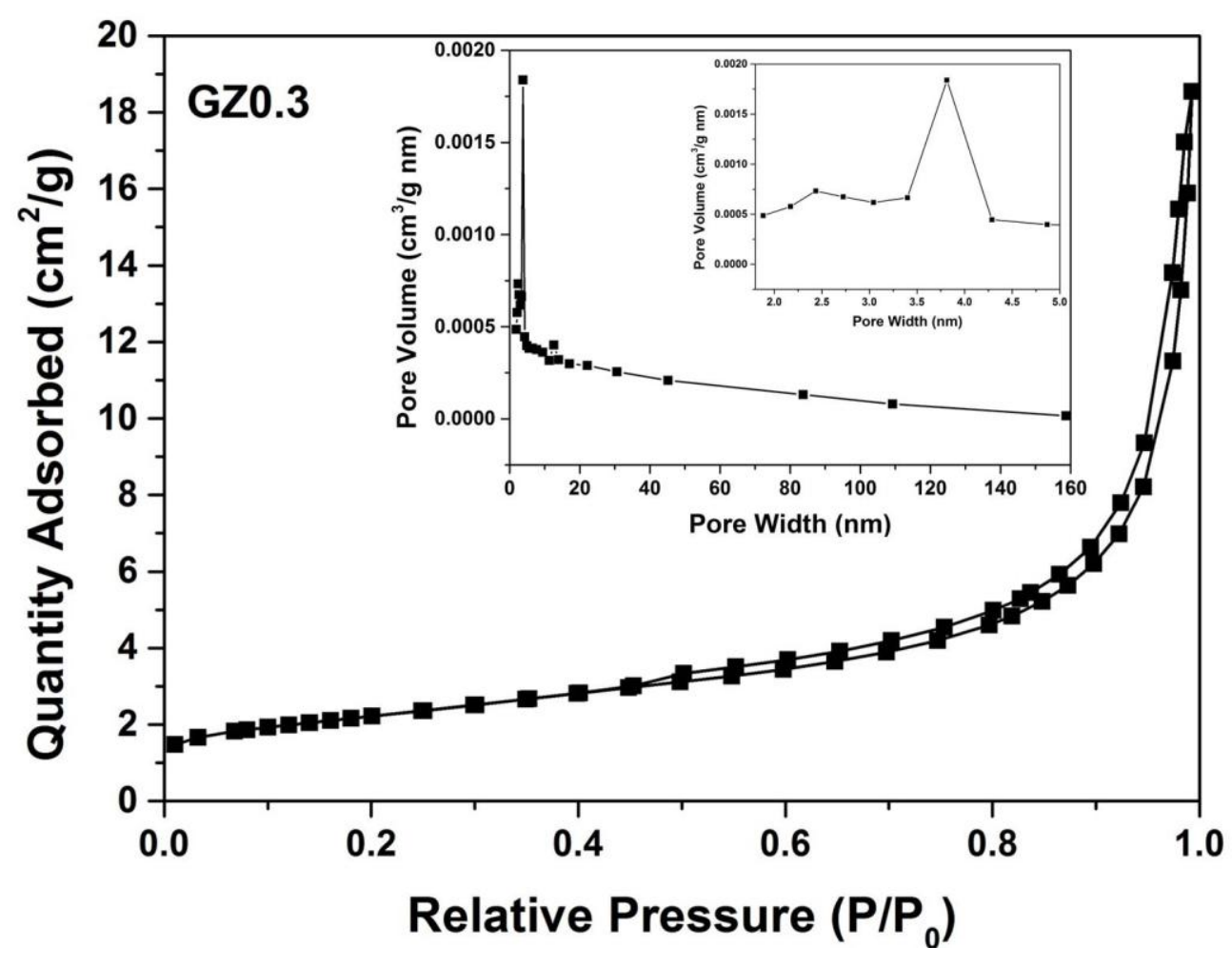

Figure 8

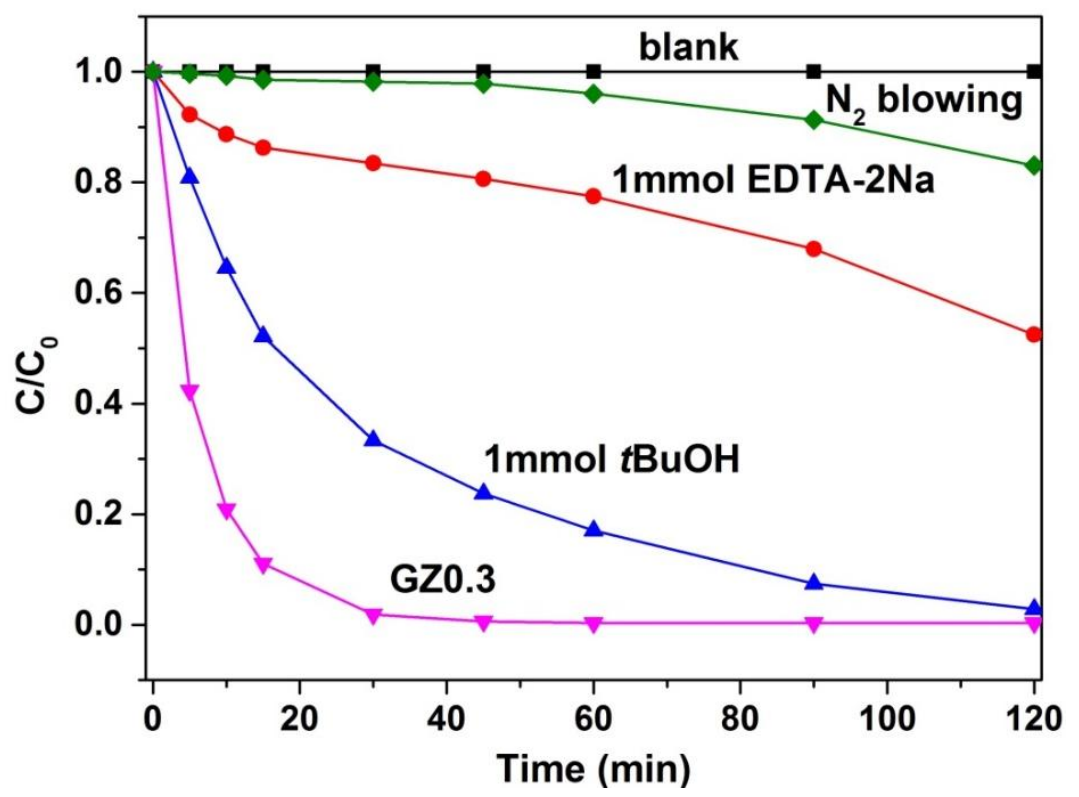

Figure 9 

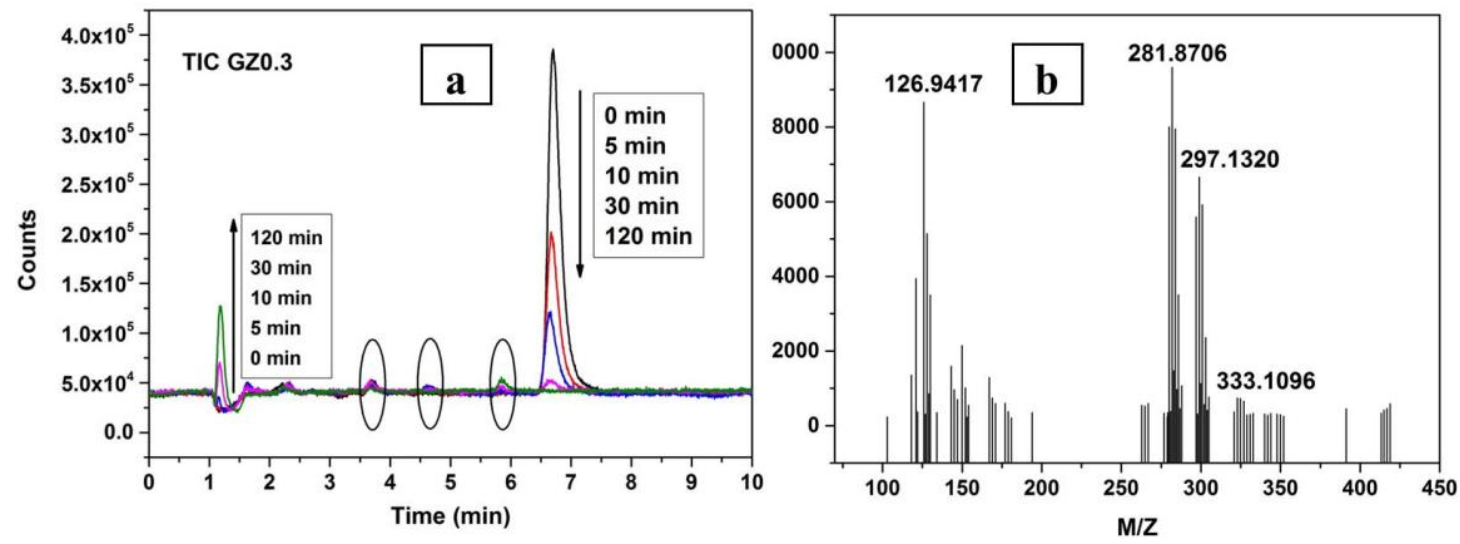

Figure 10
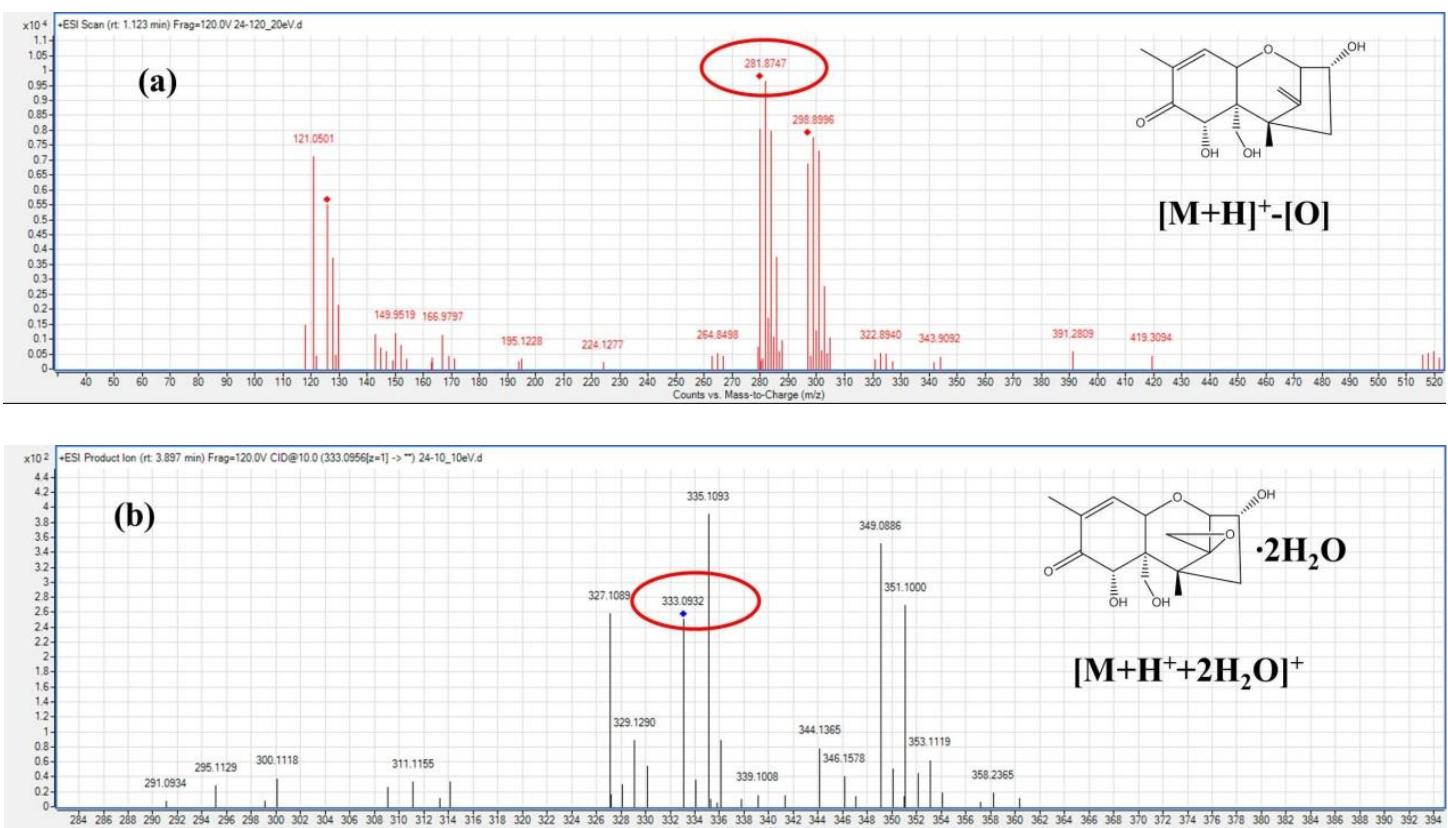
Table 1. The BET surface area and pore size distribution of GZ0.3 photocatalyst and SGH sample.

\begin{tabular}{ccccc}
\hline Sample & $\begin{array}{c}\mathbf{S}_{\mathbf{B E T}} \\
\left(\mathbf{m}^{2} / \mathbf{g}\right)\end{array}$ & $\begin{array}{c}\text { Water } \\
\text { Absorption\% }\end{array}$ & $\begin{array}{c}\text { Pore Size } \\
(\mathbf{n m})\end{array}$ & $\begin{array}{c}\text { Pore Volume } \\
\left(\mathbf{c m}^{\mathbf{3}} / \mathbf{g}\right)\end{array}$ \\
\hline GZ0.3 & 58.9217 & 85.27 & 9.0415 & 0.0178 \\
SGH & 388.9709 & 96.40 & 2.6408 & 0.2568 \\
\hline
\end{tabular}

Scheme 1. Schematic drawing illustrating synthetic route and the mechanism of charge separation and adsorption-photocatalytic process over graphene/ZnO hybrid photocatalysts under UV light irradiation.

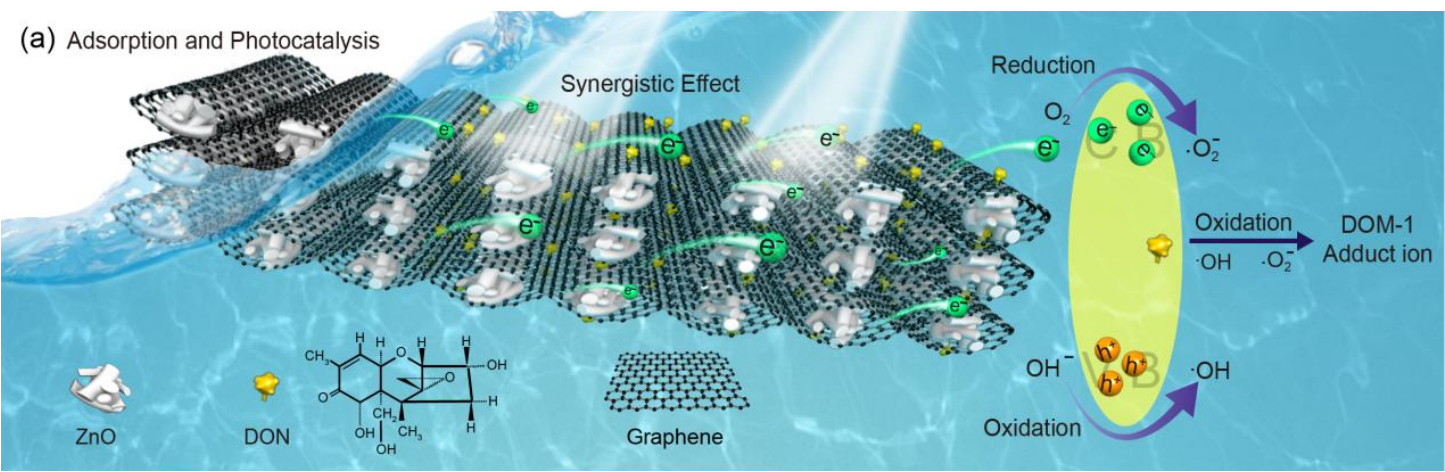

(b) Adsorption and Photocatalysis

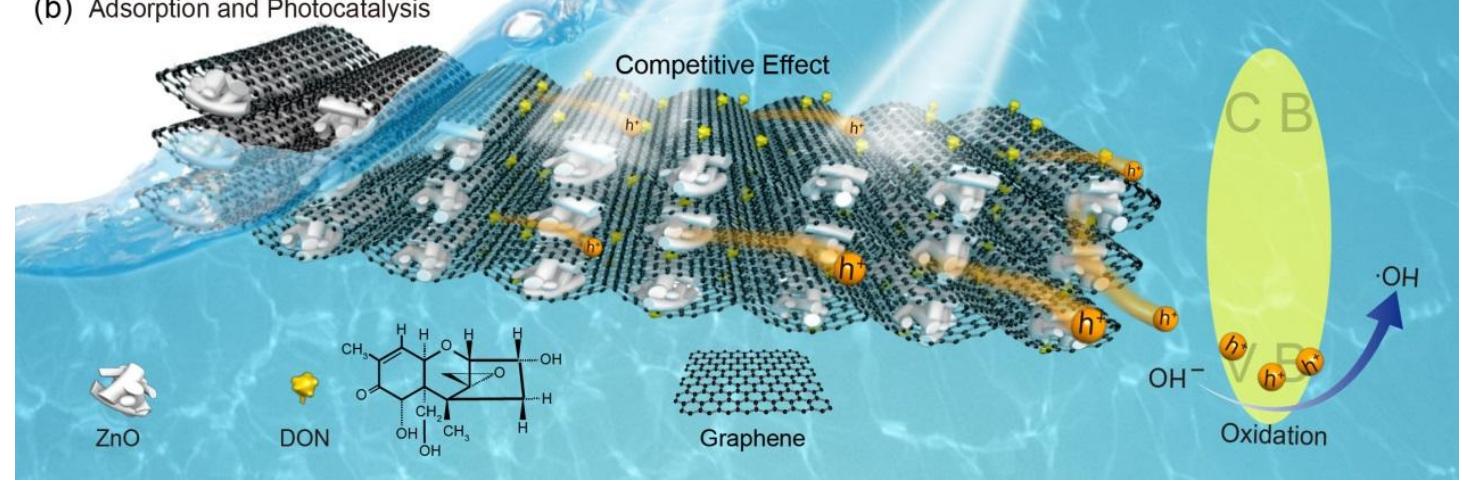

